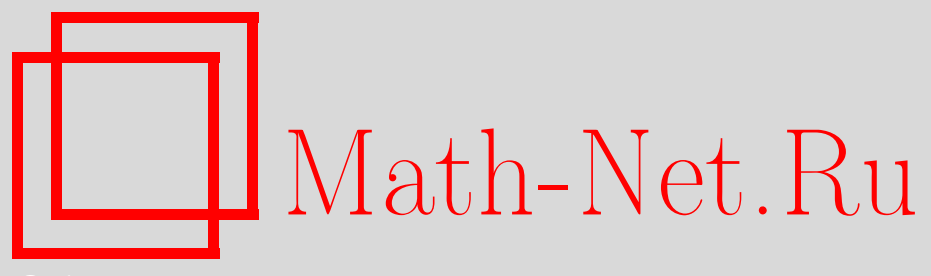

К. Гарола, С. Соццо, О физической интерпретации частичных следов: две нестандартные точки зрения, $Т M \Phi$, 2007, том 152, номер 2, 248-264

DOI: https://doi.org/10.4213/tmf6085

Использование Общероссийского математического портала Math-Net.Ru подразумевает, что вы прочитали и согласны с пользовательским соглашением http://www . mathnet.ru/rus/agreement

Параметры загрузки:

IP : 3.93 .64 .190

26 апреля 2023 г., $07: 53: 15$

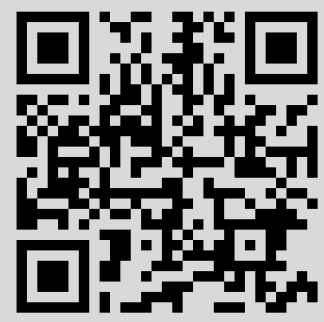




\title{
О ФИЗИЧЕСКОЙ ИНТЕРПРЕТАЦИИ ЧАСТИЧНЫХ СЛЕДОВ: ДВЕ НЕСТАНДАРТНЫЕ ТОЧКИ ЗРЕНИЯ
}

\begin{abstract}
Смешанные состояния вводятся в физике для выражения нашего неведения относительно реального состояния физической системы и представляются в стандартной квантовой механике операторами плотности. Такие операторы также появляются при рассмотрении (чистого) зацепленного состояния составной системы $\Omega$ и вычислении частичных следов представляющего ее проекционного оператора. Однако в этом случае они не представляют смешанные состояния (или собственные смеси) подсистем, а несобственные смеси, поскольку выражающие их коэффициенты в выпуклых суммах никогда не несут интерпретацию неведения. Таким образом, подсистемы составной физической системы, находящейся в зацепленном состоянии, нельзя рассматривать как состояния (задача о подсущности). Обсуждаются два альтернативных предложения: в рамках брюссельского подхода и подхода, разработанного в Лечче. Сначала суммируются основные положения первого подхода, согласно которому несобственные смеси можно рассматривать как новые чистые состояния. Затем показано, что согласно второму подходу несобственные смеси также можно рассматривать как истинные (хотя и не чистые) состояния. Несмотря на различную терминологию, эти два предложения выглядят совместимыми.
\end{abstract}

Ключевые слова: квантовая механика, несобственные смеси, задача о подсущности, брюссельский подход, Лечче-подход, семантический реализм.

\section{1. ВВЕДЕНИЕ}

В стандартной формулировке квантовой механики (KM) физическая система $\Omega$ связана с сепарабельным комплексным гильбертовым пространством $\mathcal{H}$, а состояния системы $\Omega$ представлены операторами плотности на пространстве $\mathcal{H}$, которые редуцируются к одномерным (ортогональным) проекционным операторам в случае чистых состояний. Каждый оператор плотности $W$, представляющий смешанное состояние или собственную смесъ $S$ из $\Omega$, можно выразить различными способами в виде выпуклой комбинации чистых состояний, и существует разложение

*Dipartimento di Fisica and Sezione INFN, Università del Salento, via Arnesano, 73100 Lecce, Italy. E-mail: garola@le.infn.it, sozzo@le.infn.it 
$W=\sum_{i} p_{i}\left|\psi_{i}\right\rangle\left\langle\psi_{i}\right|$, в котором каждый коэффициент $p_{i}$ представляет собой вероятность того, что $\Omega$ находится в состоянии $S_{i}$, представленном проекционным оператором $\left|\psi_{i}\right\rangle\left\langle\psi_{i}\right|$. Эта вероятность отражает наше неведение относительно реального состояния $\Omega$, а потому также и о результатах измерений, проводимых для проверки того, имеется ли у системы $\Omega$ свойство $E_{i}$, представленное с помощью $\left|\psi_{i}\right\rangle\left\langle\psi_{i}\right|$. Тем не менее если $\left\langle\psi_{i} \mid \psi_{j}\right\rangle=\delta_{i j}$, то каждое свойство $E_{i}$ является объективным в $S$ в том смысле, что его можно считать или имеющимся, или отсутствующим у $\Omega$, независимо от любых измерений. Действительно, первый случай имеет место, если реальным состоянием системы $\Omega$ является $S_{i}$, а второй - если реальным состоянием является $S_{j}$, где $j \neq i$. Теперь пусть $\Omega$ - составная система, образованная двумя подсистемами $\Omega_{1}$ и $\Omega_{2}$, в чистом зацепленном состоянии $S_{P}$, представленном проекционным оператором $|\psi\rangle\langle\psi|$. Пусть $|\psi\rangle=\sum_{i} \sqrt{p_{i}}\left|\phi_{i}(1)\right\rangle\left|\chi_{i}(2)\right\rangle$, где $p_{i} \neq 0,1$; второе неравенство выполнено, поскольку состояние $S_{P}$ является зацепленным, биортогональное разложение $|\psi\rangle$, так что

$$
|\psi\rangle\left\langle\psi\left|=\sum_{i, j} \sqrt{p_{i} p_{j}}\right| \phi_{i}(1)\right\rangle\left\langle\phi_{j}(1)|\otimes| \chi_{i}(2)\right\rangle\left\langle\chi_{j}(2)\right| .
$$

Если рассматривать только систему $\Omega_{1}$, то физическую информацию о ней, предоставляемую относительно нее КМ, можно получить вычислением частичного следа $|\psi\rangle\langle\psi|$ по отношению к системе $\Omega_{2}$, что дает $W_{1}=\operatorname{Tr}_{2}|\psi\rangle\left\langle\psi\left|=\sum_{i} p_{i}\right| \phi_{i}(1)\right\rangle\left\langle\phi_{i}(1)\right|$, и применением стандартных квантовых правил. Оператор плотности $W_{1}$ формально аналогичен $W$. Однако коэффициент $p_{i}$ в нем обозначает вероятность актуализации свойства $E_{i}(1)$ системы $\Omega$, представленной проекционным оператором $\left|\phi_{i}(1)\right\rangle \times$ $\left\langle\phi_{i}(1)\right| \otimes I_{2}$, когда бы ни состоялось измерение (или вероятность перехода из $S_{P}$ в чистое состояние, представляемое оператором $\left|\phi_{i}(1)\right\rangle\left\langle\phi_{i}(1)|\otimes| \chi_{i}(2)\right\rangle\left\langle\chi_{i}(2)\right|$, если измерение является идеальным), но он не может обозначать вероятность того, что система $\Omega_{1}$ действительно находится в состоянии $S_{i}(1)$, представляемом оператором $\left|\phi_{i}(1)\right\rangle\left\langle\phi_{i}(1)\right|$. Действительно, тогда свойство $E_{i}(1)$ должно быть объективным, как свойство $E_{i}$, рассмотренное выше, в то время как оно является необъективным в $S_{P}$, согласно стандартной интерпретации $\mathrm{KM}^{1}$ ) (т.е. нельзя считать свойство $E(1)$ ни имеющимся, ни отсутствующим у системы $\Omega$ в состоянии $S_{P}$, если измерение не проведено). На основании этого заключения можно показать, что ни одно разложение для $W_{1}$ не имеет упомянутой выше интерпретации неведения. Поэтому некоторые авторы утверждают, что $W_{1}$ представляет собой несобственную смесъ, отличая ее от собственной смеси, такой как $W$ (см., например, [4], [6], [7]).

\footnotetext{
1) Обычно считается, что необъективность представляет собой внутренне присущее и неустранимое свойство КМ в силу некоторых математических результатов, таких как теорема Белла-Кохена-Спекера [1], [2] (доказывающая контекстуальность КМ, которая означает, что результат измерения свойств физической системы в заданном состоянии не фиксирован заранее, а зависит от измерительного контекста) и теорема Белла [3] (доказывающая нелокальность КМ, что означает, что контекстуальность имеет место также и на расстоянии). Тем не менее необъективность является истинным источником большинства проблем, составляющих трудности стандартной интерпретации KM, и порождает много парадоксов и концептуальных сложностей (в частности, проблема объективизации в квантовой теории измерений, см., например, [4], [5]).
} 
Из приведенных выше аргументов следует, что в стандартной КМ операторы плотности, полученные с помощью взятия частичных следов, вообще говоря, не являются ни чистыми, ни смешанными состояниями компонентных подсистем, так что эти подсистемы никогда нельзя рассматривать как независимые сущности, что приводит к так называемой задаче о подсущности. В частности, два различных подхода к основам KM, а именно брюссельский и Лечче-подходы, непосредственно или косвенно относятся к решению этой задачи. Действительно, первый подход, разработанный в течение последних двадцати лет в Брюсселе Эртсом с сотрудниками, продолжает работу, которую начали Жош и Пирон в 60-х и 70-х годах в Женеве [8], [9] с целью получения не только физического обоснования математического аппарата КМ с помощью операциональных основ этой теории, но также для лучшего описания составных сущностей путем введения некоторых изменений в саму теорию. Лечче-подход следует другой стратегии, основываясь на критике теорем, утверждающих доказательство необъективности теории, предлагает нестандартную интерпретацию КМ (интерпретацию на основе семантического реализма), являющуюся локальной и неконтекстуальной (тем не менее сохраняющей математический аппарат КМ и его статистическую интерпретацию), с целью избежать парадоксов, поражающих стандартную KM. Тогда такой подход также предоставляет нам основные элементы для решения задачи о подсущности.

Заметим, что упомянутые выше подходы имеют некоторое количество общих черт и, по-видимому, позволяют прийти к одинаковым заключениям относительно многих проблематичных аспектов КМ [10], [11]. В частности, как брюссельский, так и Лечче-подход предполагают, что может существовать более широкая теория, включающая в себя KM, но утверждающая больше нее. Здесь, однако, мы касаемся только сравнения нестандартных предложений, возникающих из двух подходов к решению задачи о подсущности. С этой целью в разделе 2 мы кратко изложим основы брюссельского подхода, а в разделе 3 обсудим решение задачи о подсущности, возможное в его рамках, которое может вызвать крах представлений о линейности KM. Затем в разделе 4 мы кратко представим Лечче-подход, а в разделе 5 покажем, что в рамках этого подхода можно также получить решение задачи о подсущности. В обоих решениях предлагается рассматривать несобственные смеси как новые состояния физической системы, поэтому, несмотря на различную терминологию, они, по-видимому, являются совместными.

\section{2. БРЮССЕЛЬСКИЙ ПОДХОД}

В данном разделе мы даем краткое изложение основных свойств брюссельского подхода, необходимых для обсуждения исследуемого в его рамках решения задачи о подсущности [12]--[17]. Наше представление является синтетическим и частично оригинальным, поэтому мы берем на себя ответственность за возможные недостатки или неточности.

В рамках брюссельского подхода термин физическая сущность является более предпочтительным, чем стандартный широко распространенный термин физическая 
система. Пусть $\Omega$ - физическая сущность. Согласно брюссельскому подходу $\Omega$ является сущностъю свойств состояний тогда и только тогда, когда она связана с множеством состояний $\Sigma$ и с множеством свойств $\mathcal{L}$. Состояние $p$ сущности $\Omega$ интуитивно представляется "модой бытия" сущности $\Omega$. Это означает, что в каждый момент времени сущность $\Omega$ "находится" в конкретном состоянии $p \in \Sigma$. Свойство $a$ сущности $\Omega$ представляет собой атрибут сущности $\Omega$. Свойство $a$ может быть актуалъным в состоянии $p$, что означает, что $p$ таково, что $\Omega$ имеет свойство $a$ in acto, или же потенииально, что означает, что $p$ таково, что $\Omega$ не обладает свойством $a$, но может приобрести его. Таким образом, можно ввести отображение $\rho: \Sigma \times \mathcal{L} \rightarrow\{A, P\}$, где $A$ обозначает актуальное свойство, а $P$ - потенииалъное. Тогда если сущность $\Omega$ находится в состоянии $p$, то можно рассматривать множество $\xi(p)=\{a \in \mathcal{L} \mid \rho(p, a)=A\}$ всех свойств, актуальных в $p$. Аналогично для свойства $а$ сущности $\Omega$ можно рассмотреть множество $\kappa(a)=\{p \in \Sigma \mid \rho(p, a)=A\}$ всех состояний, делающих свойство а актуальным. Поэтому для любых $p \in \Sigma$ и $a \in \mathcal{L}$ выполнено $\rho(p, a)=A$ тогда и только тогда, когда $a \in \xi(p)$ или $p \in k(a)$.

Пусть $\Omega$ - сущность свойств состояний и пусть $a, b \in \mathcal{L}$. Если для любого $p \in \Sigma$ из $\rho(p, a)=A$ следует $\rho(p, b)=A$, то говорят, что $a$ влечет $b$ и пишут $a<b$ (поэтому $a<b$ тогда и только тогда, когда $\kappa(a) \subseteq \kappa(b))$. Легко видеть, что знак $<$ является отношением предпорядка на $\mathcal{L}$. Аналогично, пусть $p, q \in \Sigma$. Если для любого $a \in \mathcal{L}$ из $\rho(q, a)=A$ следует $\rho(p, a)=A$, то говорят, что $p$ по свойству влечет $q$ и пишут $p<q$ (поэтому $p<q$ тогда и только тогда, когда $\xi(q) \subseteq \xi(p))$. Легко видеть, что знак $<$ является отношением предпорядка на $\Sigma$.

Пусть для каждого множества $\mathcal{K}$ величина $\mathcal{P}(\mathcal{K})$ обозначает мощность множества $\mathcal{K}$. Приведенные выше замечания подсказывают следующее определение.

ОПРЕДЕЛЕНИЕ 1. Пусть $\Omega$ - сущность свойств состояний, характеризующаяся множеством состояний $\Sigma$, множеством свойств $\mathcal{L}$ и двумя отображениями $\xi: p \in$ $\Sigma \rightarrow \xi(p) \in \mathcal{P}(\mathcal{L})$ и $\kappa: a \in \mathcal{L} \rightarrow \kappa(a) \in \mathcal{P}(\Sigma)$, где $\xi(p)$ обозначает множество свойств, являющихся актуальными, если сущность $\Omega$ находится в состоянии $p$, а $\kappa(a)$ - множество состояний, в которых свойство а является актуальным. Для любых $p, q \in \Sigma$ и $a, b \in \mathcal{L}$ положим $p<q$ тогда и только тогда, когда $\xi(q) \subseteq \xi(p)$, а также $a<b$ тогда и только тогда, когда $\kappa(a) \subseteq \kappa(b)$, и будем говорить, что $\Omega$ описывается структурой $(\Sigma,<, \mathcal{L},<, \xi, \kappa)$ (в краткой форме $(\Sigma, \mathcal{L}, \xi, \kappa))$. Более того, мы будем говорить, что $(\Sigma, \mathcal{L}, \xi, \kappa)$ является системой свойств состояний тогда и только тогда, когда $(\mathcal{L},<)$ является полной решеткой (пересечения и объединения на которой мы обозначим как $\wedge$ и $\vee$, соответственно) и для любого $p \in \Sigma$ выполняются следующие условия.

1. Пусть $I$ и 0 - соответственно максимальный и минимальный элементы из $(\mathcal{L},<)$. Тогда $I \in \xi(p)$ и $0 \notin \xi(p)$.

2. Пусть $\left\{a_{i} \in \mathcal{L}\right\}_{i \in \mathcal{I}}-$ семейство элементов из $\mathcal{L}$. Тогда $\bigwedge_{i} a_{i} \in \xi(p)$ тогда и только тогда, когда $a_{i} \in \xi(p)$ для любого $i \in \mathcal{I}$.

Теперь, следуя Эртсу, предположим, что каждая рассматриваемая здесь физическая сущность $\Omega$ описывается системой свойств состояний. Тогда можно доказать, 
что стандартный формализм КМ можно воспроизвести путем добавления подходящих аксиом, относящихся к системе свойств состояний $(\Sigma, \mathcal{L}, \xi, \kappa)$. Для начала соберем все требуемые аксиомы в следующее определение.

ОПРЕДЕЛЕНИЕ 2 . Пусть $(\Sigma, \mathcal{L}, \xi, \kappa)$ - система свойств состояний и пусть $\mathcal{A}$ - множество всех атомов решетки $(\mathcal{L},<)$. Тогда:

1. $(\Sigma, \mathcal{L}, \xi, \kappa)$ удовлетворяет аксиоме определения состояния, если для любых $p, q \in \Sigma$ из $\bigwedge_{a \in \xi(p)} a=\bigwedge_{b \in \xi(q)} b$ следует $p=q$.

2. $(\Sigma, \mathcal{L}, \xi, \kappa)$ удовлетворяет аксиоме атомарности, если для любого $p \in \Sigma$ элемент $\bigwedge_{a \in \xi(p)}$ a является атомом решетки $(\mathcal{L},<)$.

3. $(\Sigma, \mathcal{L}, \xi, \kappa)$ удовлетворяет аксиоме ортодополнительности, если решетка $(\mathcal{L},<)$ является ортодополняемой, т.е. существует отображение ' $: \mathcal{L} \rightarrow \mathcal{L}$ такое, что для любых $a, b \in \mathcal{L}$ из $\left(a^{\prime}\right)^{\prime}=a, a \wedge a^{\prime}=0, a \vee a^{\prime}=I$ и из $a<b$ следует $b^{\prime}<a^{\prime}$.

4. $(\Sigma, \mathcal{L}, \xi, \kappa)$ удовлетворяет аксиоме закона накрытия, если для любых $a, x \in \mathcal{L}$ и $b \in \mathcal{A}$ из $a<x<a \vee b$ следует или $x=a$, или $x=a \vee b$.

5. $(\Sigma, \mathcal{L}, \xi, \kappa)$ удовлетворяет аксиоме слабой модулярности, если решетка $(\mathcal{L},<)$ является ортодополняемой и для любого $a, b \in \mathcal{L}$ из $a<b$ следует $\left(b \wedge a^{\prime}\right) \vee a=b$.

6. $(\Sigma, \mathcal{L}, \xi, \kappa)$ удовлетворяет аксиоме плоской транзитивности, если для любых $s, t \in \mathcal{A}$ существуют два различных атома $s_{1}, s_{2} \in \mathcal{A}$ и автоморфизм $f$ на $\mathcal{L}$ такой, что $t=f(s)$ и для любого $a \in\left[0, s_{1} \vee s_{2}\right]$ выполнено $f(a)=a$.

7. $(\Sigma, \mathcal{L}, \xi, \kappa)$ удовлетворяет аксиоме неприводимости, если решетка $(\mathcal{L},<)$ является ортодополняемой и для любого $b \in \mathcal{L}$ из $b=(b \wedge a) \vee\left(b \wedge a^{\prime}\right) \forall a \in \mathcal{L}$ следует или $b=0$, или $b=I$.

8. $(\Sigma, \mathcal{L}, \xi, \kappa)$ удовлетворяет аксиоме бесконечной длины, если решетка $(\mathcal{L},<)$ является ортодополняемой и содержит бесконечное множество взаимно ортогональных элементов (где $b, c \in \mathcal{L}$ взаимно ортогональны тогда и только тогда, когда существует такое свойство $a \in \mathcal{L}$, что $b<a$ и $\left.c<a^{\prime}\right)$.

Теперь можно доказать следующую теорему о представлении.

Теорема. Пусть $\Omega$ - физическая сущность, описываемая системой свойств состояний $(\Sigma, \mathcal{L}, \xi, \kappa)$, удовлетворяющей всем аксиомам из определения 2. Тогда решетка $(\mathcal{L},<)$ изоморфна полной решетке $(\mathcal{L}(\mathcal{H}), \subseteq)$ проекционных операторов на бесконечномерном вещественном, комплексном или кватернионном гильбертовом пространстве $\mathcal{H}$. Атомы решетки $(\mathcal{L}(\mathcal{H}), \subseteq)$, т.е. одномерные проекциоеные операторы на $\mathcal{H}$, находятся во взаимно однозначном соответствии с атомами решетки $(\mathcal{L},<)$ и с элементами множества $\Sigma^{2)}$. Ортодополняемость индуцирована ортогоналъностъю структуры пространства $\mathcal{H}$.

Приведенная выше теорема о представлении позволяет воспроизвести стандартный квантовый формализм из операциональной структуры, называемой системой

\footnotetext{
2) Заметим, что существование взаимно однозначного отображения множества $\Sigma$ на $\mathcal{A}$ следует из аксиомы атомарности. В рамках брюссельского подхода эта аксиома иногда заменяется более слабой аксиомой (см., например, [15]): в формулировке теоремы вместо множества $\Sigma$ нужно подставить подмножество $\Lambda$ всех атомов решетки $(\Sigma,<)$ (или атомных состояний). Здесь из соображений простоты мы выбрали более сильное утверждение.
} 
свойств состояний и введенной с помощью брюссельского подхода. Задача доказательства этой теоремы привлекала большое число ученых, а ее решение положило конец исследованиям так называемой задачи координатизации [18]. Доказательство теоремы длинное и сложное и требует использования целого ряда важных математических результатов, таких как теорема Пирона [9], фундаментальная теорема проективной геометрии [19], теорема Солера [20] и теорема Глисона [21]. Окончательный результат является актуальным, поскольку он дает (частичное) операциональное подтверждение стандартного формализма KM, который в этом случае представляется более строго обоснованным. Однако тогда возникает новая проблема. Действительно, хорошо известно, что если мы захотим применить КМ к составным сущностям, мы столкнемся с целым рядом трудностей. Поэтому мы посвящаем следующий раздел именно этому вопросу.

\section{3. ЗАДАЧА О ПОДСУЩНОСТИ В РАМКАХ БРЮССЕЛЬСКОГО ПОДХОДА}

Для обсуждения задачи о подсущности нам следует предварительно сделать замечание относительно теоремы, сформулированной в разделе 2. Подчеркнем, что эта теорема, строго говоря, устанавливает взаимно однозначное соответствие между атомами решетки $(\mathcal{L}(\mathcal{H}), \subseteq)$ и элементами множества $\Sigma$, откуда следует, что $\Sigma$ не содержит смешанных состояний. Поэтому все аксиомы из определения 2 относятся к системе свойств состояний, для которой рассматриваются только чистые состояния. Это согласуется с интуитивным представлением о состоянии как о "моде бытия" сущности $\Omega$. Согласно брюссельскому подходу реальность квантовой сущности в КМ выражается чистым состоянием, представленным одномерным проекционным оператором в соответствующем гильбертовом пространстве. Собственные смеси очевидным образом были учтены в рамках брюссельского подхода, но они не классифицируются как моды бытия сущности, поскольку представляют не реальность сущности, а отсутствие нашего знания относительно этой реальности. Действительно, появляющиеся в них вероятности выражают (как и в стандартной интерпретации KM, см. раздел 1) наше субъективное неведение об актуальногом состоянии сущности [14].

Перейдем к определению подсущности в соответствии с брюссельским подходом.

Во-первых, пусть $\Omega$ и $\Omega^{\prime}$ - две физические сущности, описываемые с помощью системы свойств состояний $(\Sigma, \mathcal{L}, \xi, \kappa)$ и $\left(\Sigma^{\prime}, \mathcal{L}^{\prime}, \xi^{\prime}, \kappa^{\prime}\right)$, соответственно. Если $\Omega$ - подсущность в $\Omega^{\prime}$, то разумно спросить, верно ли, что если $\Omega^{\prime}$ находится в состоянии $p^{\prime}$, то $\Omega$, как часть $\Omega^{\prime}$, находится в состоянии $m\left(p^{\prime}\right)$. Отображение $m$, введенное таким образом, должно быть сюръективным (каждое состояние сущности $\Omega$ соответствует по крайней мере одному состоянию сущности $\Omega^{\prime}$ ), но необязательно инъективным (различные состояния $\Omega^{\prime}$ могут соответствовать одному и тому же состоянию $\Omega$ ).

Во-вторых, если $\Omega$ - подсущность в $\Omega^{\prime}$, то каждое свойство $а$ сущности $\Omega$ должно соответствовать свойству $n(a)$ сущности $\Omega^{\prime}$. Отображение $n$, введенное таким образом, должно быть инъективным (если два свойства сущности $\Omega$ различны, то они 
также различны, если рассматриваются как свойства сущности $\left.\Omega^{\prime}\right)$, но необязательно сюръективным (имеются свойства сущности $\Omega^{\prime}$, не соответствующие свойствам сущности $\Omega$ ).

Наконец, в-третьих, если свойство $а$ является актуальным, когда $\Omega$ находится в состоянии $m\left(p^{\prime}\right)$, то свойство $n(a)$ должно быть актуальным, когда $\Omega^{\prime}$ находится в состоянии $p^{\prime}$ (приниип ковариантности, см., например, [15]).

Согласно приведенным выше аргументам брюссельский подход дает определение подсущности, которое можно обобщить следующим образом.

ОПРЕДЕЛЕНИЕ 3 . Пусть $\Omega$ и $\Omega^{\prime}$ - две физические сущности, описываемые с помощью систем свойств состояний $(\Sigma, \mathcal{L}, \xi, \kappa)$ и $\left(\Sigma^{\prime}, \mathcal{L}^{\prime}, \xi^{\prime}, \kappa^{\prime}\right)$, соответственно. Мы будем говорить, что $\Omega$ является подсущностъю в $\Omega^{\prime}$ тогда и только тогда, когда существует сюръективное отображение $m: p^{\prime} \in \Sigma^{\prime} \rightarrow m\left(p^{\prime}\right) \in \Sigma$ и инъективное отображение $n: a \in \mathcal{L} \rightarrow n(a) \in \mathcal{L}^{\prime}$ такие, что для любых $p^{\prime} \in \Sigma^{\prime}$ и $a \in \mathcal{L}$ мы имеем $a \in \xi\left(m\left(p^{\prime}\right)\right)$ тогда и только тогда, когда $n(a) \in \xi^{\prime}\left(p^{\prime}\right)$.

Теперь рассмотрим КМ. Согласно теореме о представлении все элементы системы свойств состояний, удовлетворяющие аксиомам из определения 2 , можно описать в рамках стандартного математического аппарата KM. В частности, понятие подсущности в KM можно выразить, если в качестве $\Sigma$ взять множество всех атомов решетки $(\mathcal{L}(\mathcal{H}), \subseteq)$, а в качестве $\mathcal{L}$ - множество всех проекционных операторов решетки $(\mathcal{L}(\mathcal{H}), \subseteq)$. Тогда можно доказать, что подсистемы составной системы (или сущности, в соответствии с терминологией брюссельского подхода) не являются подсущностями в смысле определения 3. Действительно, пусть $\Omega$ и $\Omega^{\prime}$ - две квантовые сущности, связанные с гильбертовыми пространствами $\mathcal{H}$ и $\mathcal{H}^{\prime}$, соответственно. Предположим, что $\Omega$ является подсистемой в $\Omega^{\prime}$, так что $\mathcal{H}^{\prime}=\mathcal{H} \otimes \mathcal{G}$, где $\mathcal{G}$ - другое гильбертово пространство. Чистое состояние сущности $\Omega$ представляет собой одномерный проекционный оператор $P_{\psi}$ на $\mathcal{H}$, а чистое состояние сущности $\Omega^{\prime}-$ одномерный проекционный оператор $P_{\psi^{\prime}}^{\prime}$ на $\mathcal{H}^{\prime}$. Более того, все свойства подсистемы $\Omega$ представляются с помощью проекционного оператора $P$ на $\mathcal{H}$ и соответствуют тем свойствам $\Omega^{\prime}$, которые представляются с помощью проекционного оператора $P^{\prime}=P \otimes \mathbb{I}_{\mathcal{G}}$, где $\mathbb{I}_{\mathcal{G}}$ - тождественный оператор на $\mathcal{G}$. С учетом замечаний, сделанных в начале данного раздела, и стандартных результатов, упомянутых в разделе 1 , можно сделать вывод, что в этом случае отображение $m$ из определения 3 не может существовать. Действительно, соответствие между чистым состоянием $p^{\prime}$ для $\Omega^{\prime}$ и чистым состоянием $m\left(p^{\prime}\right)$ для $\Omega$, удовлетворяющее условиям определения 3 , можно получить тогда и только тогда, когда $p^{\prime}$ является произведением состояний. Таким образом, $\Omega$ не является подсущностью в $\Omega^{\prime}$ в смысле определения 3 , что доказывает наше утверждение.

Полученный выше результат показывает, что стандартный квантовый формализм не может непротиворечивым образом описывать физические сущности, составленные из подсущностей. Тем не менее представляется разумным допустить, что сущности такого типа существуют. Таким образом, мы получаем задачу о подсущности: 
можно ли дополнить формализм КМ так, чтобы сущности и подсущности можно было прямо описывать в рамках расширенного формализма?

Согласно Эртсу [14], [15] задача о подсущности была известна еще на раннем этапе развития КМ, но терялась за смятением, которое порой присутствует в литературе при описании собственных и несобственных смесей. Действительно, во многих статьях и учебниках по КМ (см., например, [8], [22]) написано, что даже в случае, когда составная система находится в чистом нефакторизованном состоянии, подсистемы находятся в смешанных состояниях, а чистые состояния отсутствуют, а именно: смешанные состояния, представленные с помощью операторов плотности, получаются путем вычисления частичных следов. На первый взгляд кажется, что утверждение о том, что подсистемы, хотя и не находящиеся в чистом состоянии, находятся по крайней мере в смешанном состоянии, предлагает частичное решение задачи о подсущности в КМ. Но это утверждение неверно, поскольку в нем пренебрегается концептуальным различием между собственными и несобственными смесями, на которое мы указывали в разделе 1.

Решение задачи о подсущности, предлагаемое в рамках брюссельского подхода, которое мы здесь обсуждаем ${ }^{3)}$, является достаточно радикальным и состоит в том, что несобственные смеси интерпретируются как чистые состояния в рамках более общих допущений, при которых дополненные квантовые сущности занимают место введенных выше квантовых сущностей. Мы не будем воспроизводить здесь трактовку брюссельского подхода, поскольку это потребовало бы введения слишком большого количества предварительных понятий. Вместо этого мы предложим новую трактовку, которая, как нам представляется, воспроизводит главное предложение брюссельского подхода (в рамках которого понятие дополненной квантовой сущности является, однако, более общим). Для этой цели введем следующее определение.

ОПРЕДЕЛЕНИЕ 4 . Пусть $\Omega$ - физическая сущность. Мы будем говорить, что $\Omega$ является дополненной квантовой сущностъю тогда и только тогда, когда описывающая его система свойств состояний $(\Sigma, \mathcal{L}, \xi, \kappa)$ допускает каноническое представление на сепарабельном комплексном гильбертовом пространстве $\mathcal{H}$, причем:

1) $(\Sigma,<)$ изоморфно с сохранением порядка предупорядоченному множеству $(\mathcal{W}(\mathcal{H}),<)$ всех операторов плотности на $\mathcal{H}$ (здесь знак $<$ обозначает как предпорядок $\Sigma$, так и канонический математический предпорядок на $\mathcal{W}(\mathcal{H})$, индуцированный включением областей значения);

2) $(\mathcal{L},<)$ изоморфно с сохранением порядка полной решетке $(\mathcal{L}(\mathcal{H}), \subseteq)$ всех проекционных операторов на $\mathcal{H}$;

3) смешанные состояния сущности $\Omega$ представлены выпуклыми суммами операторов плотности в $\mathcal{W}(\mathcal{H})$ (а следовательно, по-прежнему операторами плотности).

Сопоставление определения 4 со сформулированной выше теоремой показывает, что со стандартной квантовой точки зрения дополненная квантовая сущность

3) Заметим, что в рамках брюссельского подхода это решение не является единственным. Альтернативное решение действительно обсуждалось в ряде работ Эртса с сотрудниками (см., например, [16]), однако является достаточно условным и в настоящей работе не представляет для нас интереса. 
характеризуется более широким множеством чистых состояний, нежели квантовая сущность, поскольку каждый оператор плотности представляет чистое состояние (в рамках брюссельского подхода чистые состояния, которые представляются операторами плотности, не сводимыми к проекционным операторам, также называются состояниями плотности). Введение дополненных квантовых сущностей дает очевидное решение задачи о подсущности. Действительно, когда дополненная составная квантовая сущность $\Omega^{\prime}$ находится в чистом состоянии $p_{W}$, представленном оператором плотности $W$, вычисление частичных следов от $W$ дает операторы плотности, которые можно интерпретировать как операторы, представляющие чистые состояния компонентных подсистем. Тогда интуитивно очевидно, и это можно доказать математически [14], что все условия из определения 3 удовлетворяются, если $\Omega$ является компонентной подсистемой в $\Omega^{\prime}$, так что $\Omega$ является подсущностью в $\Omega^{\prime}$, и тогда задача о подсущности пропадает.

Дополнение KM, предложенное выше для решения задачи о подсущности (которое можно рассматривать как шаг в поиске решения более общей задачи - задачи такого обобщения KM, что и разделенные квантовые сущности можно будет описывать с помощью новой теории [12], [13], [17]), ставит несколько новых интересных вопросов. Кратко опишем некоторые из них.

1. Пусть $\Omega$ - дополненная квантовая сущность, а $p_{W}$ - состояние плотности сущности $\Omega$, представленное оператором $W$. Тогда $W$ можно выразить различными способами как выпуклую сумму проекционных операторов. Появляющиеся в сумме коэффициенты нельзя интерпретировать как вероятности выражения нашего неведения относительно реального состояния сущности $\Omega$, как это имеет место, когда $W$ представляет собственную смесь в стандартной КМ. Однако их можно интерпретировать как вероятности актуализации частных свойств или как переходные вероятности, как в случае несобственных смесей в стандартной КМ (см. раздел 1). Это подчеркивает недостатки математического формализма, используемого в теории, а именно модели гильбертова пространства. Действительно, в рамках этого формализма состояния, имеющие различные физические интерпретации, представляются одинаковыми математическими объектами.

2. Более общим образом, из представления смешанных состояний, введенного в определении 4, следует, что каждое смешанное состояние представляется с помощью оператора плотности на $\mathcal{H}$, что даже усугубляет проблему, отмеченную в пункте 1. Согласно Эртсу, если принять, что множество чистых состояний физической сущности представлено множеством всех операторов плотности, то это множество уже не может быть представлено в линейном пространстве, что может вызвать крах принципа суперпозиции. Поэтому решение задачи о подсущности в рамках брюссельского подхода “ставит на карту линейность КМ" [17].

3. Можно предположить, что вероятностный переход из чистого состояния в смешанное, происходящий всегда, когда проводится измерение, следует стандартным квантовым правилам (формула Людерса), откуда следует, что введенные состояния плотности невозможно эмпирически отличить от смешанных состояний, представленных теми же операторами плотности. Это может благоприятствовать мнению, 
что решение задачи о подсущности в рамках брюссельского подхода теоретически интересно, но эмпирически несущественно. Однако хорошо известно, что операторы плотности, полученные взятием частичных следов, в случае составной сущности в зацепленном состоянии не эволюционируют унитарно. Это предполагает, что состояния плотности могут эволюционировать другими способами, нежели смешанные состояния, представленные теми же операторами плотности. Поэтому Эртс пишет [16]: “Если бы мы могли экспериментально реализовать нелинейную эволюцию одной из подсущностей, которая приведена в зацепленное состояние с другой подсущностью, как подсущности объединенной сущности, то было бы возможно проверить нашу гипотезу и экспериментально выяснить, являются ли плотности состояний чистыми или смешанными состояниями".

4. Операциональное подтверждение стандартного формализма KM, кратко обобщенное в разделе 2 , должно быть модифицировано при рассмотрении дополненных квантовых сущностей. Точнее говоря, система свойств состояний, описывающая сущность такого типа, не может удовлетворять всем аксиомам из определения 2, поскольку в этом случае не должно существовать плотности состояния. С учетом наших предыдущих замечаний из пункта 2 мы приходим к тому, что следует подвергнуть сомнению аксиому, из которой следуют линейность и принцип суперпозиции, а именно аксиому закона накрытия ${ }^{4)}$. Выяснение всех этих вопросов, однако, выходит за рамки настоящей работы.

\section{4. ЛЕЧЧЕ-ПОДХОД}

Решение задачи о подсущности в рамках брюссельского подхода, представленное в разделе 3, интересно, однако обладает целым рядом недостатков. В частности, введение состояний как "мод бытия" сущности может выглядеть "метафизическим" и раздражать многих прагматически настроенных физиков. С другой стороны, такое представление состояния не является теоретически несущественным, поскольку из него следует предположение о том, что сюръективное отображение из определения 3 существует, что могло бы побудить некоторых ученых отказаться от определения 3 и решить, что подсущности в определенном здесь смысле просто не существуют (такая альтернатива серьезно рассматривалась в самом брюссельском подходе, см. сноску 3). Поэтому, как, забегая вперед, мы отмечали в разделе 1, мы желаем сравнить решение из раздела 3 с другим решением, которое можно получить в рамках Лечче-подхода [23]-[31]. В данном разделе мы подытожим некоторые основные особенности Лечче-подхода, которые потребуются нам для достижения нашей цели.

1. Физическая сущность $\Omega$ связана с множеством П приготовляющих устройств и с множеством $\mathcal{R}$ регистрирующих устройств, характеризующих ее. Приготовляющее устройство $\pi \in \Pi$, будучи построено и активировано, выполняет приготовление индивидуального образца сущности $\Omega$ (физического обгекта). Регистрирующее

${ }^{4)}$ Интересно отметить, что если мы хотим получить обобщение КМ, которое могло бы описывать разделенные квантовые сущности, то и аксиома слабой модулярности тоже “ставится на карту" [12], [13], [17]. 
устройство $r \in \mathcal{R}$, будучи построено и активировано после приготовления, выполняет регистрацию и выдает на выходе один из двух возможных результатов: "да" и "нет".

2. Всякая реальная ситуация локализована в некоторой физической лаборато$p u и j$, где все приготовления и все регистрации можно повторять в различные моменты времени. Вообще говоря, $j$ можно рассматривать как пространственно-временную область. Обозначим множество всех лабораторий через $J$. Приготовляющее устройство $\pi \in \Pi$ можно активировать множество раз в любой лаборатории для приготовления ансамблей физических объектов. Если каждый физический объект в каждом ансамбле тестируется с помощью устройства $r \in \mathcal{R}$ сразу после приготовления, то для каждого ансамбля на выходе мы получим некоторую частоту результатов “да" (или "нет"). Теперь предположим, что приготовляющее и регистрирующее устройства выбраны так, что для каждой пары $(\pi, r)$ эти частоты приближаются к пределу, когда рассматриваются ансамбли с возрастающим числом физических объектов, причем этот предел одинаков в каждой лаборатории.

3. Пусть $\pi_{1}, \pi_{2} \in \Pi$ и пусть $\Phi_{1}$ и $\Phi_{2}$ - два ансамбля физических объектов, приготовленных с помощью устройств $\pi_{1}$ и $\pi_{2}$, соответственно, в лаборатории $j$. Мы будем говорить, что приготовляющие устройства $\pi_{1}$ и $\pi_{2}$ физически эквивалентнъ тогда и только тогда, когда каждое регистрирующее устройство $r \in \mathcal{R}$ при применении к физическим объектам из $\Phi_{1}$ и $\Phi_{2}$ дает на выходе результат "да" с частотами, приближающимися к одному и тому же пределу (при возрастающем числе физических объектов) для обоих ансамблей и в каждой лаборатории. Такое соотношение физической эквивалентности канонически индуцирует разбиение множества П. Мы будем называть состояниями элементы этого разбиения и ниже будем обозначать множество всех состояний через $\mathcal{S}$. Отметим, что такое операциональное определение не различается для чистых и смешанных состояний.

4. Можно рассмотреть множество всех физических объектов, приготовленных в лаборатории $j$ с помощью приготовляющих устройств, принадлежащих П: это множество называется областью определения $D_{j}$ лаборатории $j$.

5. Можно рассмотреть в области определения $D_{j}$ лаборатории $j$ подмножество всех физических объектов, приготовленных с помощью многократно активированного приготовляющего устройства $\pi$ : такое множество является расширением $\rho_{j}(\pi)$ состояния $\pi$ в лаборатории $j$. Тогда расширение $\rho_{j}(S)$ состояния $S$ в $j$ определяется как объединение всех $\rho_{j}(\pi)$, где $\pi \in S$.

6. Пусть $r_{1}, r_{2} \in \mathcal{R}$ и пусть $\Phi_{1}$ и $\Phi_{2}-$ два ансамбля физических объектов, приготовленных с помощью одного и того же приготовляющего устройства $\pi$ в лаборатории $j$. Мы будем говорить, что $r_{1}$ и $r_{2}$ являются физически эквивалентными тогда и только тогда, когда при применении к физическим объектам из ансамблей $\Phi_{1}$ и $\Phi_{2}$, соответственно, они дают на выходе результат “да" с частотами, приближающимися к одному и тому же пределу (при возрастающем числе физических объектов) для обоих ансамблей и в каждой лаборатории, каким бы ни было приготовляющее устройство $\pi$. Это соотношение физической эквивалентности канонически индуцирует разбиение множества $\mathcal{R}$. Мы будем называть эффектами элементы этого раз- 
биения. Внутри множества всех эффектов можно выбрать подмножество точных эффектов или свойств, элементы которых являются классами идеальных регистрирующих устройств. Ниже мы будем обозначать множество всех свойств через $\mathcal{E}$.

7. Предположим, что в области определения $D_{j}$ лаборатории $j$ определено подмножество всех физических объектов, которые дадут на выходе результат “да" при тестировании с помощью заданного регистрирующего устройства $r$ сразу после приготовления: это будет расширением $\rho_{j}(r)$ устройства $r$ в $j$. Более того, пусть $r_{1}$ и $r_{2}-$ регистрирующие устройства. При $\rho_{j}\left(r_{1}\right)=\rho_{j}\left(r_{2}\right)$ в каждой лаборатории $j$ устройства $r_{1}$ и $r_{2}$ являются очевидным образом эквивалентными в смысле пункта 6 , но обратное утверждение не выполняется априори. Поэтому вводится дополнительное предположение, что устройства $r_{1}$ и $r_{2}$ являются эквивалентными тогда и только тогда, когда для любой лаборатории $j \in J$ имеет место $\rho_{j}\left(r_{1}\right)=\rho_{j}\left(r_{2}\right)$.

8. Используя предположение, сделанное в пункте 7, можно ввести для любых $E \in \mathcal{E}$ и $j \in J$ расширение $\rho_{j}(E)$ свойства $E$ в $j$, полагая $\rho_{j}(E)=\rho_{j}(r)$, где $r \in E$.

9. Для любой лаборатории $j \in J$ расширения $\rho_{j}\left(S_{1}\right)$ и $\rho_{j}\left(S_{2}\right)$ двух различных состояний $S_{1}$ и $S_{2}$ должны иметь пустое пересечение, поскольку объекты в $\rho_{j}\left(S_{1}\right)$ приготовлены с помощью устройств, которые не эквивалентны устройствам, с помощью которых приготовляются объекты в $\rho_{j}\left(S_{2}\right)$. С другой стороны, поскольку каждый физический объект в $D_{j}$ приготовлен с помощью некоторого приготовляющего устройства (см. пункт 4), множество расширений всех возможных состояний в любой лаборатории $j$ должно исчерпать область определения $D_{j}$. Другими словами, множество $\mathcal{S}$ всех состояний индуцирует для каждого $j \in J$ разбиение области $D_{j}$. Отсюда следует, в частности, что два состояния $S_{1}$ и $S_{2}$ совпадают тогда и только тогда, когда для каждого $j \in J$ имеет место $\rho_{j}\left(S_{1}\right)=\rho_{j}\left(S_{2}\right)$. Более того, если мы введем различие между чистыми и "нечистыми" состояниями (см. ниже раздел 5 , пункт 2), то расширения чистого и "нечистого" состояний никогда не пересекутся, в чем и заключается существенное отличие Лечче-подхода от других подходов, например, подхода Людвига [32], к основам КМ.

10. Мы будем говорить, что физический объект в состоянии $S$ заведомо дает на выходе результат "да" для каждого регистрирующего устройства по свойству $E$ тогда и только тогда, когда для каждой лаборатории $j \in J$ выполнено $\rho_{j}(S) \subseteq \rho_{j}(E)$. Поэтому для любых $S \in \mathcal{S}$ и $E \in \mathcal{E}$ можно ввести заведомо истинную областъ $\mathcal{E}_{t}(S)$ состояния $S$ и область $\mathcal{S}_{y}(E)$ с заведомым результатом “да" свойства $E$, которые определяются следующим образом:

$$
\begin{aligned}
& \mathcal{E}_{t}(S)=\left\{E \in \mathcal{E} \mid \text { для любой } j \in J, \quad \rho_{j}(S) \subseteq \rho_{j}(E)\right\}, \\
& \mathcal{S}_{y}(E)=\left\{S \in \mathcal{S} \mid \text { для любой } j \in J, \quad \rho_{j}(S) \subseteq \rho_{j}(E)\right\} \text {. }
\end{aligned}
$$

Очевидно, для любых $S \in \mathcal{S}$ и $E \in \mathcal{E}$ выполнено $E \in \mathcal{E}_{t}(S)$ тогда и только тогда, когда $S \in \mathcal{S}_{y}(E)$. Более того, если мы положим, что для любых $E, F \in \mathcal{E}$ выполнено $E \leqslant F$ тогда и только тогда, когда $\mathcal{S}_{y}(E) \subseteq \mathcal{S}_{y}(F)$, и для любых $S, T \in \mathcal{S}$ выполнено $S \leqslant T$ тогда и только тогда, когда $\mathcal{E}_{t}(T) \subseteq \mathcal{E}_{t}(S)$, то мы получим, что $(\mathcal{E}, \leqslant)$ и $(\mathcal{S}, \leqslant)$ представляют собой предупорядоченные множества. 
11. Из определений в пункте 10 следует, что сущность $\Omega$, характеризуемая множествами $\mathcal{S}$ и $\mathcal{E}$ и отображениями $\mathcal{E}_{t}: \mathcal{S} \rightarrow \mathcal{E}_{t}(\mathcal{S}) \subseteq \mathcal{P}(\mathcal{E})$ и $\mathcal{S}_{y}: \mathcal{E} \rightarrow \mathcal{S}_{y}(\mathcal{E}) \subseteq \mathcal{P}(\mathcal{S})$, где $\mathcal{P}(\mathcal{E})$ и $\mathcal{P}(\mathcal{S})$ обозначают мощности множеств $\mathcal{E}$ и $\mathcal{S}$, соответственно, представляет собой сущность свойств состояний в смысле, установленном в разделе 2. Далее мы будем предполагать, что структура $\left(\mathcal{S}, \mathcal{E}, \mathcal{E}_{t}, \mathcal{S}_{y}\right)$ также является системой свойств состояний (конечно, это свойство $\left(\mathcal{S}, \mathcal{E}, \mathcal{E}_{t}, \mathcal{S}_{y}\right)$ можно получить как следствие более слабого допущения в рамках Лечче-подхода; из соображений краткости мы не будем останавливаться на этом вопросе).

Таким образом, в рамках Лечче-подхода мы воспроизвели структуру, являющуюся основной в рамках брюссельского подхода. Но описанный выше формализм вводит (см. пункт 8) характерную особенность, которая является типичной для Лечче-подхода, но не появляется ни в рамках брюссельского подхода, ни в стандартной интерпретации $\mathrm{KM},-$ объективность свойств. Если расширение $\rho_{j}(E)$ определяется в каждой лаборатории $j$ для каждого свойства $E$, то результат регистрации, выполненный регистрирующим устройством из $E$, примененный к физическому объекту $x$, не зависит от измерительного контекста (он равен "да" тогда и только тогда, когда $\left.x \in \rho_{j}(E)\right)$. В семантических терминах можно сказать, что истинное значение утверждения вида $E(x)$, приписывающего свойство $E$ физическому объекту $x$, определяется семантически $(E(x)$ истинно тогда и только тогда, когда $\left.x \in \rho_{j}(E)\right)$, независимо от какой-либо теоретической или экспериментальной процедуры, которая может привести к знанию о нем, а потому также и в тех физических ситуациях, в которых физическая теория (в нашем случае КМ) утверждает, что невозможно теоретически предсказать или эмпирически достичь знания о нем с помощью подходящих измерений. В семантическом смысле, избегающем любых онтологических привязок, согласно Лечче-подходу свойства являются объективными (поэтому вытекающая отсюда интерпретация КМ была названа семантическим реализмом, или СР-интерпретацией). Конечно, из объективности свойств следует, что СР-интерпретация приходит в столкновение со стандартной интерпретацией, которая вместо этого утверждает необъективность свойств на основании эмпирических (например, эксперимента с двумя щелями) или теоретических (например, упомянутых в сноске 1 теорем) аргументов. Поэтому одновременно с разработкой СР-интерпретации был проведен тщательный анализ этих аргументов, при этом в каждом их них выявилась некоторая слабость [25], [29], [30]. В частности, оказалось, что теоретические аргументы в пользу необъективности основаны на неявных допущениях, которые становятся довольно сомнительными, если сделать их явными. Действительно, эти допущения противостоят эпистемологической точке зрения, предполагающей состоятельность эмпирических квантовых законов и в тех физических ситуациях, относительно которых сама КМ утверждает, что их в принципе невозможно проверить. Если такую точку зрения подвергнуть критике ${ }^{5)}$, то

\footnotetext{
5) Напомним, что критика основана на новом эпистемологическом взгляде, согласно которому теоретические законы любой физической теории рассматриваются как математические схемы, из которых можно вывести эмпирические законы. Предполагается, что эмпирические законы имеют силу во всех тех физических ситуациях, в которых их можно экспериментально проверить, в то время как нельзя сделать допущения об их состоятельности в тех физических ситуациях, в которых
} 
необъективность свойств проявляется как выбор интерпретации, а не как логическая необходимость, и станут возможными альтернативные интерпретации. Среди них СР-интерпретация восстанавливает объективность, не требуя никаких изменений в математическом аппарате KM.

\section{5. ЗАДАЧА О ПОДСУЩНОСТИ В РАМКАХ ЛЕЧЧЕ-ПОДХОДА}

Важнейшая черта, характерная для СР-интерпретации, заключающаяся в объективности свойств (введеная для того, чтобы избежать ряда проблем и парадоксов, вытекающих из стандартной интерпретации КM; в частности, отметим среди них трудности в квантовой теории измерений [30]), предполагает естественное решение задачи о подсущности, удивительным образом похожее на решение, предлагаемое в рамках другого, а именно брюссельского подхода. Обсудим это, снова действуя шаг за шагом.

1. Состояния и свойства имеют различные операциональные определения (см. пункты 3 и $\mathbf{6}$ раздела 4), а их расширения имеют различные свойства (см. пункты $\mathbf{5}$, 8 и 9 раздела 4), откуда следует, что их необходимо тщательно различать с физической точки зрения (такое различие также имеет место в рамках брюссельского подхода). В частности, можно никогда не распознать неизвестное состояние физического объекта $x$ с помощью процедуры регистрации, в точности как в стандартной $\mathrm{KM}$, где идеальное измерение помещает $x$ в конечное состояние, но предоставляет только ограниченную информацию о начальном состоянии объекта $x$. Наоборот, из объективности свойств следует, что можно получить информацию о том, обладает или не обладает $x$ свойством $E$, используя регистрирующее устройство $r \in E$ (конечно, $x$ обладает свойством $E$ тогда и только тогда, когда регистрация дает на выходе результат “да"), что не согласуется со стандартной КМ, где идеальное измерение актуализирует измеряемое свойство, о котором, вообще говоря, неизвестно, обладал или не обладал им объект $x$ до измерения.

2. Из пункта 1 следует, что в рамках Лечче-подхода вероятность нахождения заданного результата при проведении измерения физического объекта $x$ можно интерпретировать как наше неведение относительно свойств, которыми обладает $x$ (в этом смысле можно сказать, что оно эпистемично), каким бы ни было состояние физического объекта. Различие между чистыми и "нечистыми" состояниями можно по-прежнему ввести на основании различных значений вероятностей свойств в этих состояниях, но не различных интерпретаций (эпистемологичных или нет) самих вероятностей. В частности, можно принять стандартное представление состояний

некоторый общий принцип запрещает их проверку (такая позиция является непротиворечивой, в частности, при операциональном и антиметафизическом отношении к стандартной КМ). В классической физике новая точка зрения не вызывает никаких существенных изменений, поскольку нет такой физической ситуации, в которой эмпирический закон не мог бы в принципе быть проверен. Наоборот, если в КМ заданы граничные или начальные условия, приписывающие физической системе (более точно, образцу системы) несовместимые свойства, то строится гипотеза, согласно которой такая физическая ситуация не может быть получена эмпирически, поэтому в такой ситуации можно не делать допущений о состоятельности эмпирических законов, выведенных из общего формализма KM. Странно, но этого нового взгляда оказывается достаточно, чтобы лишить силы доказательство теорем, упомянутых в сноске 1. 
через операторы плотности и характеризовать чистые состояния как состояния, для которых представляющие их операторы плотности сводятся к проекционным операторам.

3. В пункте 2 мы тщательно избегали характеризовать "нечистые" состояния как смеси. Для понимания причины такого выбора вспомним, что в пункте $\mathbf{3}$ раздела 4 каждое состояние интерпретировалось в операциональных терминах как класс эквивалентности приготовляющих устройств. Если рассматривать состояние $S$, представленное оператором плотности $\sum_{i} p_{i}\left|\psi_{i}\right\rangle\left\langle\psi_{i}\right|$, то ансамбль физических объектов в состоянии $S$ можно реализовать посредством смешивающего приготовляющего устройства, т.е. устройства, которое смешивает физические объекты, приготовленные с помощью устройств, принадлежащих состояниям $S_{1}, S_{2}, \ldots$, представленным соответствующими проекционными операторами $\left|\psi_{1}\right\rangle\left\langle\psi_{1}|,| \psi_{2}\right\rangle\left\langle\psi_{2}\right|, \ldots$. В этом случае коэффициент $p_{i}$ не может быть интерпретирован только как в пункте 2 , но также как вероятность того, что данный физический объект из ансамбля объектов в состоянии $S$ действительно находится в состоянии $S_{i}$. Тем не менее согласно Лечче-подходу нет очевидных физических причин, чтобы предполагать, что $S$ содержит только смешивающие приготовляющие устройства. Если отказаться от этого допущения, то рассмотрение $S$ как смешанного состояния приведет к заблуждению.

4. Из пункта 3 следует, что в случае составных физических систем можно принять, что операторы плотности, полученные вычислением частичных следов, представляют состояния, в которых также имеются приготовления, не смешивающие в том смысле, как это определено в пункте 3. Следует ли эти состояния рассматривать как чистые (как в брюссельском подходе) или как “нечистые” (как в пункте 3) предмет соглашения.

5. Теперь можно использовать брюссельское определение подсущности в рамках Лечче-подхода, просто заменив в определении $3(\Sigma, \mathcal{L}, \xi, \kappa)$ и $\left(\Sigma^{\prime}, \mathcal{L}^{\prime}, \xi^{\prime}, \kappa^{\prime}\right)$ на $\left(\mathcal{S}, \mathcal{E}, \mathcal{E}_{t}, \mathcal{S}_{y}\right)$ и $\left(\mathcal{S}^{\prime}, \mathcal{E}^{\prime}, \mathcal{E}_{t}^{\prime}, \mathcal{S}_{y}^{\prime}\right)$, соответственно. Тогда пусть $\Omega$ и $\Omega^{\prime}$ - две квантовые сущности, связанные с гильбертовыми пространствами $\mathcal{H}$ и $\mathcal{H}^{\prime}$ и описываемые с помощью систем свойств состояний $\left(\mathcal{S}, \mathcal{E}, \mathcal{E}_{t}, \mathcal{S}_{y}\right)$ и $\left(\mathcal{S}^{\prime}, \mathcal{E}^{\prime}, \mathcal{E}_{t}^{\prime}, \mathcal{S}_{y}^{\prime}\right)$, соответственно. Более того, предположим, что $\Omega$ является подсистемой системы $\Omega^{\prime}$, так что $\mathcal{H}^{\prime}=\mathcal{H} \otimes \mathcal{G}$ (где $\mathcal{G}$ также является гильбертовым пространством), и обозначим через $S_{W}, E_{P}$, $S_{W^{\prime}}^{\prime}, E_{P^{\prime}}^{\prime}$ соответственно состояние сущности $\Omega$, представленное оператором плотности $W$ на $\mathcal{H}$, свойство сущности $\Omega$, представленное проекционным оператором $P$ на $\mathcal{H}$, состояние сущности $\Omega^{\prime}$, представленное оператором плотности $W^{\prime}$ на $\mathcal{H}^{\prime}$, и свойство сущности $\Omega^{\prime}$, представленное проекционным оператором $P^{\prime}$ на $\mathcal{H}^{\prime}$. Легко доказать, что отображения $m: S_{W^{\prime}}^{\prime} \in \mathcal{S}^{\prime} \rightarrow S_{W} \in \mathcal{S}$ такое, что $W=\operatorname{Tr}_{\mathcal{G}} W^{\prime}$ (где $\operatorname{Tr}_{\mathcal{G}}$ - частичный след оператора плотности $W^{\prime}$ относительно подсущности, связанной с гильбертовым пространством $\mathcal{G})$, и $n: E_{P} \in \mathcal{E} \rightarrow E_{P^{\prime}}^{\prime} \in \mathcal{E}^{\prime}$, причем $P^{\prime}=P \otimes \mathbb{I}_{\mathcal{G}}$ $\left(\right.$ где $\mathbb{I}_{\mathcal{G}}$ - тождество в $\left.\mathcal{G}\right)$, удовлетворяют условиям из определения 3 . Таким образом, $\Omega$ является подсущностью сущности $\Omega^{\prime}$ и задача о подсущности оказывается решенной в рамках Лечче-подхода. 
Приведенное выше решение можно сравнить с решением, предложенным в разделе 3. Тогда становится ясно, что оба решения совместны, если не считать соглашения, определяющего класс чистых состояний. Этот результат кажется нам очень интересным, поскольку он повышает надежность обоих решений и может привести к более тщательному рассмотрению предложений о расширении стандартной КМ, следующих из брюссельского и Лечче-подходов.

Остается отметить, что вероятностное определение состояний в пункте $\mathbf{3}$ раздела 4 (которое обычно используется в литературе по основам КМ и не является специфическим для Лечче-подхода [18]) в случае "нечистых" состояний собирает вместе смешивающие и несмешивающие приготовляющие устройства, которые поэтому невозможно различить с помощью измерений. Это объясняет глубокие корни проблемы неразличимости, упомянутой в пункте $\mathbf{3}$ раздела 3. Более того, это, возможно, позволит объяснить тот факт, что для одного и того же оператора плотности могут иметь место как унитарная, так и неунитарная эволюции (см. пункт 3 раздела 3). Действительно, предлагается различать смешивающие и несмешивающие приготовляющие устройства, вводя новые соотношения эквивалентности на множестве П, строго содержащиеся в физических соотношениях эквивалентности, определенных в пункте 3 раздела 4. Таким образом, каждое состояние $S$ должно быть связано с семейством скрытых состояний (посредством чего, по-видимому, вводится нечто вроде теории скрытых переменных; однако имеются некоторые важные особенности, которые мы не имеем возможности здесь обсуждать, см., например, [31]). Они должны быть эквивалентны относительно измерений, но могли бы вести себя по-разному относительно временно́й эволюции.

Сформулированное выше предложение представляется особенно подходящим для завершения данной работы. Действительно, оно естественным образом поддерживает предложения Эртса, упомянутые в пункте $\mathbf{3}$ раздела 3, что снова демонстрирует схожесть брюссельского и Лечче-подходов, несмотря на их знаменательные различия.

\section{Список литературы}

[1] J.S. Bell, Rev. Mod. Phys., 38 (1966), 447.

[2] S. Kochen, E. P. Specker, J. Math. Mech., 17 (1967), 59.

[3] J. S. Bell, Physics, 1 (1964), 195.

[4] P. Busch, P. J. Lahti, P. Mittelstaedt, The Quantum Theory of Measurement, Lecture Notes in Phys. New Ser. m. Monogr., 2, Springer, Berlin, 1991.

[5] P. Busch, A. Shimony, Stud. Hist. Philos. Sci. B. Stud. Hist. Philos. Modern Phys., 27 (1996), 397.

[6] K.-E. Hellwig, K. Kraus, Commun. Math. Phys., 11 (1969), 214.

[7] B. D'Espagnat, Conceptual Foundations of Quantum Mechanics, Math. Phys. Monogr. Ser., 20, Benjamin, Reading, MA, 1976.

[8] J. M. Jauch, Foundations of Quantum Mechanics, Addison-Wesley, Reading, MA, 1968.

[9] C. Piron, Foundations of Quantum Physics, Benjamin, Reading, MA, 1976.

[10] C. Garola, J. Pykacz, S. Sozzo, Found. Phys., 36 (2006), 862.

[11] S. Sozzo, Lecce and Brussels: two proposals for a realistic and objective interpretation of quantum mechanics, PhD Thesis, Univ. Lecce, Lecce, 2006. 
[12] D. Aerts, The one and the many: towards a unification of the quantum and the classical description of one and many physical entities, PhD Thesis, Brussels Free Univ., Brussels, 1981.

[13] D. Aerts, Found. Phys., 12 (1982), 1131.

[14] D. Aerts, Internat. J. Theoret. Phys., 38 (1999), 289.

[15] D. Aerts, "Quantum mechanics: structures, axioms and paradoxes", Quantum Structures and the Nature of Reality, Einstein Meets Magritte, 7, eds. D. Aerts, J. Pykacz, Kluwer, Dordrecht, 1999, 141.

[16] D. Aerts, Internat. J. Theoret. Phys., 39 (2000), 483.

[17] D. Aerts, F. Valckenborgh, "The linearity of quantum mechanics at stake: the description of separated quantum entities", Probing the Structure of Quantum Mechanics. Nonlinearity, Nonlocality, Computation and Axiomatics (Brussels, Belgium, 2000), eds. D. Aerts, M. Czachor, T. Durt, World Scientific, Singapore, 2002, 20.

[18] E. G. Beltrametti, G. Cassinelli, The Logic of Quantum Mechanics, Encyclopedia Math. Appl., 15, Addison-Wesley, Reading, MA, 1981.

[19] V. Varadarajan, Geometry of Quantum Theory, vol. I, Nostrand, Princeton-TorontoLondon, 1968; vol. II, Nostrand, New York-Toronto-London, 1970.

[20] M. P. Solèr, Comm. Algebra, 23 (1995), 219.

[21] A. M. Gleason, J. Math. Mech., 6 (1957), 885.

[22] C. Cohen-Tannoudji, B. Diu, F. Laloë, Quantum Mechanics, Wiley, New York, 1977.

[23] C. Garola, Internat. J. Theoret. Phys., 30 (1991), 1.

[24] C. Garola, "Against "paradoxes": a new quantum philosophy for quantum mechanics", Quantum Structures and the Nature of Reality, Einstein Meets Magritte, 7, eds. D. Aerts, J. Pykacz, Kluwer, Dordrecht, 1999, 103.

[25] C. Garola, Found. Phys., 30 (2000), 1539.

[26] C. Garola, Found. Phys., 32 (2002), 1597.

[27] C. Garola, Found. Phys. Lett., 16 (2003), 605.

[28] C. Garola, L. Solombrino, Found. Phys., 26 (1996), 1121.

[29] C. Garola, L. Solombrino, Found. Phys., 26 (1996), 1329.

[30] C. Garola, J. Pykacz, Found. Phys., 34 (2004), 449.

[31] C. Garola, Internat. J. Theoret. Phys., 44 (2005), 807.

[32] G. Ludwig, Foundations of Quantum Mechanics, vol. I, Texts Monogr. Phys., Springer, New York, 1983. 